\title{
Prognostic and predictive significance of long interspersed nucleotide element-1 methylation in advanced-stage colorectal cancer
}

Mami Kaneko ${ }^{1,2,3^{*}}$, Masanori Kotake ${ }^{1}$, Hiroyuki Bando ${ }^{2}$, Tetsuji Yamada ${ }^{2}$, Hirofumi Takemura ${ }^{1}$ and Toshinari Minamoto ${ }^{3}$

\begin{abstract}
Background: Hypomethylation of Long Interspersed Nucleotide Element-1 (LINE-1) is associated with worse prognosis in colorectal cancer (CRC). However, little is known about the relevance of this marker for the prognosis and response to chemotherapy of metastatic and recurrent (advanced-stage) CRC. Our aim was therefore to investigate whether tumor LINE-1 hypomethylation correlates with patient survival and with response to 5fluorouracil (5-FU)/ oxaliplatin (FOLFOX) chemotherapy in advanced-stage CRC.

Methods: The study included 40 CRC patients who developed metastasis or local recurrence after surgery and subsequently underwent FOLFOX therapy. Progression-free and overall survival were estimated using the KaplanMeier method. LINE-1 methylation levels in formalin-fixed and paraffin-embedded primary tumor tissues were measured by MethyLight assay and correlated with patient survival. In vitro analyses were also conducted with human colon cancer cell lines having different LINE-1 methylation levels to examine the effects of 5-FU and oxaliplatin on LINE-1 activity and DNA double-strand-breaks.
\end{abstract}

Results: Patients with LINE-1 hypomethylation showed significantly worse progression-free (median: 6.6 vs 9.4 months; $P=0.02$ ) and overall (median: 16.6 vs 23.2 months; $P=0.01$ ) survival following chemotherapy compared to patients with high methylation. LINE-1 hypomethylation was an independent factor for poor prognosis $(P=0.018)$ and was associated with a trend for non-response to FOLFOX chemotherapy. In vitro analysis showed that oxaliplatin increased the LINE-1 score in LINE-1-expressing (hypomethylated) cancer cells, thereby enhancing and prolonging the effect of 5FU against these cells. This finding supports the observed correlation between tumor LINE-1 methylation and response to chemotherapy in CRC patients.

Conclusions: Tumor LINE-1 hypomethylation is an independent marker of poor prognosis in advanced-stage CRC and may also predict non-response to combination FOLFOX chemotherapy. Prospective studies are needed to optimize the measurement of tumor LINE-1 methylation and to confirm its clinical impact, particularly as a predictive marker.

Keywords: LINE-1 elements, Oxaliplatin, Colorectal cancer, Prognosis, Metastasis

\footnotetext{
* Correspondence: kanekomami@ipch.jp; campy_mk@yahoo.co.jp

${ }^{1}$ Department of General and Cardiothoracic Surgery, Graduate School of

Medical Science, Kanazawa University, 13-1 Takara-machi, Kanazawa

920-8641, Japan

${ }^{2}$ Department of Gastrointestinal Surgery, Ishikawa Prefectural Central

Hospital, Kanazawa, Japan

Full list of author information is available at the end of the article
}

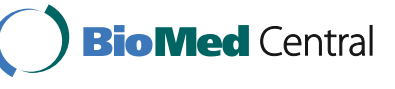

(c) The Author(s). 2016 Open Access This article is distributed under the terms of the Creative Commons Attribution 4.0 International License (http://creativecommons.org/licenses/by/4.0/), which permits unrestricted use, distribution, and reproduction in any medium, provided you give appropriate credit to the original author(s) and the source, provide a link to the Creative Commons license, and indicate if changes were made. The Creative Commons Public Domain Dedication waiver (http://creativecommons.org/publicdomain/zero/1.0/) applies to the data made available in this article, unless otherwise stated. 


\section{Background}

Colorectal cancer (CRC) is one of the most common cancer types worldwide and was responsible for an estimated 694,000 deaths in 2012 [1]. Although the incidence of CRC is increasing, mortality from CRC has decreased in many countries [1]. This trend is likely due to early diagnosis and the development of multidisciplinary treatments [2]. Chemotherapy with 5-fluorouracil (5-FU) has been the key drug for the last 50 years. Untreated patients with metastatic CRC have a median survival period of only 8 months, whereas this is prolonged to $16.2-19.5$ months by treatment with 5 -FU and leucovorin in combination with oxaliplatin or irinotecan $[3,4]$.

Aberrant hypermethylation of tumor DNA is thought to contribute to the development and progression of cancer, including CRC, by silencing the expression of tumor suppressor genes [5]. On the other hand, genome-wide hypomethylation of tumor DNA induces genomic instability by reactivating transposable DNA sequences and this change has also been associated with colorectal carcinogenesis [6-8]. Emerging evidence indicates that epigenetic mechanisms such as aberrant DNA methylation can trigger resistance to 5-FU, oxaliplatin and irinotecan in CRC [9]. Oxaliplatin exerts its cytotoxic effects via DNA damage and the arrest of nucleic acid synthesis [10]. The different mechanisms of resistance to oxaliplatin include histone methylation and various gene alterations. However, the most important mechanism appears to involve the DNA mismatch repair (MMR) and nucleotide excision repair (NER) systems $[11,12]$. Although tumor DNA hypermethylation has been implicated in chemoresistance [13, 14], little is known about its relationship to oxaliplatin resistance.

Long interspersed nucleotide element-1 (LINE-1) is one of the retrotransposons that are distributed throughout the genome. LINE-1 is about $6 \mathrm{~kb}$ long, occupies approximately $18 \%$ of the genome and plays a role in regulating genomic structure and function $[15,16]$. Its insertion into gene promoters and exons results in the functional disruption of these sequences, while its insertion into introns causes exon skipping, alternative splicing and transcriptional attenuation [17]. Because of the high frequency of LINE-1 in the genome, its hypomethylation provides an accurate representation of global DNA hypomethylation $[8,18]$. Tumor LINE-1 hypomethylation has consistently been associated with worse survival in CRC patients [19-21]. We also reported earlier that LINE-1 hypomethylation was predictive of good response to oral fluoropyrimidines in the adjuvant setting [22] and that LINE-1 methylation levels in primary tumors correlated well with those of metastatic lesions from the same patient [23, 24]. Recently, the predictive and prognostic significance of LINE-1 hypomethylation has been studied by several independent groups in metastatic (Stage IV) CRC using large patient cohorts $[25,26]$. Furthermore, the potential association of LINE1 hypomethylation with therapeutic efficacy of FOLFOX (combined folate, 5-fluorouracil and oxaliplatin) regimen has also been reported [27]. However, so far there are no systemic studies on the prognostic and predictive significance of LINE-1 hypomethylation in metastatic CRC patients who undergo treatment with FOLFOX. The aim of the present study was therefore to determine whether the level of LINE-1 methylation in the primary tumor of advanced-stage CRC patients was associated with overall survival and with response to FOLFOX combination chemotherapy. We also investigated whether LINE-1 methylation levels in colon cancer cell lines were associated with sensitivity to 5 -FU or oxaliplatin.

\section{Methods}

\section{CRC patients and tissue samples}

This study included 40 patients with CRC who underwent surgery between October 1999 and November 2010 at the Ishikawa Prefectural Central Hospital (clinical details shown in Table 1). Surgical specimens including primary tumor were fixed by neutralized formalin and embedded in paraffin for routine histopathology diagnosis. Between 2005 and 2011, patients who developed metastasis or unresectable local recurrence after surgery were treated with a FOLFOX regimen consisting of bolus intravenous injection with oxaliplatin $\left(85 \mathrm{mg} / \mathrm{m}^{2}\right)$, leucovorin $\left(200 \mathrm{mg} / \mathrm{m}^{2}\right)$ and 5 -FU $\left(400 \mathrm{mg} / \mathrm{m}^{2}\right)$, followed by continuous infusion over $46 \mathrm{~h}$ with 5 -FU $\left(2,400 \mathrm{mg} / \mathrm{m}^{2}\right)$ [28]. This regimen was repeated every 2 weeks until disease progression or the occurrence of intolerable toxicities. Response to the FOLFOX regimen was evaluated over 4 to 6 courses of treatment according to the Response Evaluation Criteria in Solid Tumors (RECIST version 1.1) [29]. None of the patients received 5-FU-based chemotherapy before primary surgery. Progression-free survival (PFS), overall survival (OS) and 5-year overall probability of survival (OPS) were all defined from the time of initiation of chemotherapy.

Tumor tissue was identified in representative $10 \mu \mathrm{m}$ thick paraffin sections. Following deparaffinization the genomic DNA was isolated using QIAamp DNA mini kits (Qiagen, Hilden, Germany).

\section{Colon cancer cell lines}

Colon cancer cell lines SW480, HCT116, Caco-2 and RKO were obtained from the American Type Culture Collection (Manassas, VA, USA). The cells were maintained at $37{ }^{\circ} \mathrm{C}$ with $5 \% \mathrm{CO}_{2}$ in Dulbecco's modified Eagle's medium (DMEM) supplemented with $10 \%$ fetal bovine serum and antibiotics (100 units/mL penicillin G, $100 \mu \mathrm{g} /$ $\mathrm{mL}$ streptomycin; Gibco, Grand Island, NY, USA). 
Table 1 Association between the tumor LINE-1 methylation levels and clinicopathological characteristics of the advancedstage CRC patients

\begin{tabular}{|c|c|c|c|c|}
\hline & $n$ & \multicolumn{2}{|c|}{ LINE-1 methylation } & $p$-value \\
\hline \multicolumn{5}{|l|}{ Gender } \\
\hline Male & 26 & 46.8 & $(39.8-53.2)$ & \multirow[t]{2}{*}{0.738} \\
\hline Female & 15 & 48.5 & $(43.6-53.0)$ & \\
\hline \multicolumn{5}{|l|}{ Age (years) } \\
\hline $65 \geq$ & 20 & 47.4 & $(43.1-51.7)$ & \multirow[t]{2}{*}{0.806} \\
\hline$>65$ & 21 & 47.4 & $(41.4-52.6)$ & \\
\hline \multicolumn{5}{|l|}{ Sites of primary tumors } \\
\hline Colon & 25 & 47.2 & $(42.2-51.7)$ & \multirow[t]{2}{*}{0.517} \\
\hline Rectum & 16 & 47.7 & $(43.0-54.2)$ & \\
\hline \multicolumn{5}{|l|}{ Tumor histological types } \\
\hline Well-differentiated & 4 & 47.6 & $(45.6-52.9)$ & \multirow[t]{3}{*}{0.941} \\
\hline Moderately-differentiated & 34 & 47.5 & $(42.5-53.2)$ & \\
\hline Poorly-differentiated & 3 & 46.7 & $(41.5-52.9)$ & \\
\hline \multicolumn{5}{|l|}{ Status } \\
\hline Advanced (stage IV) & 18 & 45 & $(42.1-48.9)$ & \multirow[t]{2}{*}{0.113} \\
\hline Recurrence & 23 & 49.3 & $(43.3-54.4)$ & \\
\hline \multicolumn{5}{|l|}{ Distant metastasis } \\
\hline One organ & 29 & 48.4 & $(43.8-54.0)$ & \multirow[t]{2}{*}{0.185} \\
\hline Multiple organs & 12 & 45 & $(40.7-51.4)$ & \\
\hline \multicolumn{5}{|l|}{ Previous treatment with 5-FU } \\
\hline Yes & 22 & 48.8 & $(42.5-53.2)$ & \multirow[t]{2}{*}{0.429} \\
\hline No & 19 & 45.8 & $(42.6-51.7)$ & \\
\hline \multicolumn{5}{|l|}{ Number of previous regimens } \\
\hline 0 & 30 & 46.8 & $(43.5-52.3)$ & \multirow[t]{2}{*}{0.612} \\
\hline 1 & 11 & 49.1 & $(40.1-55.8)$ & \\
\hline
\end{tabular}

LINE-1 methylation levels are shown as the median value (25th-75th percentile). Histological type of the primary tumor was classified into wellmoderately- and poorly-differentiated adenocarcinoma according to their grading. 5-FU, 5-fluorouracil; LINE-1, long interspersed nucleotide element-1; $\mathrm{n}$, number of patients

\section{Drug treatment of cells}

We determined the concentration of oxaliplatin $(2 \mu \mathrm{M})$ for the treatment of colon cancer cells by reference to the reported $\mathrm{IC}_{50}$ (concentration resulting in $50 \%$ growth inhibition) [30]. The concentration of 5 -FU $(1$ or $2 \mu \mathrm{M})$ was determined in our previous study [22]. Cells were cultured for $24 \mathrm{~h}$ and then treated with oxaliplatin for $2 \mathrm{~h}$. Following replacement of the medium, cells were treated with 5 -FU for $48 \mathrm{~h}$ or $120 \mathrm{~h}$. The effects of drug treatment were examined by measuring LINE-1 activity and DNA double-strand breaks as described below.

\section{LINE-1 methylation analysis}

Genomic DNA was extracted from formalin-fixed and paraffin-embedded (FFPE) tumor tissues and cultured cells and treated with bisulfite using the EpiTect Plus
Bisulfite Conversion kit (QIAGEN). LINE-1 methylation in CRC tumor tissues was measured by using the MethyLight assay as described previously [31]. LINE-1 methylation in CRC cell lines was quantified using methylation-specific real-time PCR [32] with the ABI-PRISM 7900 Sequence Detection System (Applied Biosystems, Osaka, Japan) and Premix Ex Taq (TaKaRa Bio, Otsu, Japan). The primers and probes used for LINE-1 methylation analyses [23, 32] were shown in Additional file 1: Table S1 and Additional file 2: Figure S1. The level of LINE-1 methylation was expressed as a median value with 25 th-75th percentile. LINE-1 methylation levels for the colon cancer cells used in this study were determined in our previous study [22].

\section{Formaldehyde-assisted isolation of regulatory elements (FAIRE)}

FAIRE isolates DNA regions with active chromatin and therefore enriches in functional genomic elements such as active promoters and transcriptional start sites. This was performed as described earlier [33] to enrich and measure the active promoter of LINE-1 in colon cancer cells. It is reported that DNA without histone binding isolated by FAIRE is active in retrotransposons including LINE-1 [34]. Following treatment of cells with $1 \%$ formaldehyde for $5 \mathrm{~min}$, glycine was added to a final concentration of $125 \mathrm{mM}$ for $5 \mathrm{~min}$. The cross-linked chromatin was sheared by sonication and this was followed by phenolchloroform extraction. Covalently linked protein-DNA complexes were sequestered into the organic phase, leaving protein-free DNA fragments in the aqueous phase. The DNA was precipitated by incubation with sodium acetate, glycogen and ethanol at $-20{ }^{\circ} \mathrm{C}$ overnight. The amount of LINE-1 was measured by quantitative real-time PCR as described above and the LINE-1 score was obtained using the $\Delta \Delta \mathrm{Ct}$ method. Design of the primers used for the real-time PCR was shown in Additional file 1: Table S1 and Additional file 2: Figure S1.

\section{Measurement of phospho-histone H2A.X ( $\gamma \mathrm{H} 2 \mathrm{~A} . \mathrm{X})$ foci}

Cells were plated onto coverslips for $24 \mathrm{~h}$ prior to treatment with drugs as described above. Following treatment, cells were fixed in $4 \%$ paraformaldehyde for $10 \mathrm{~min}$ at room temperature and permeabilized for 5 min in $0.2 \%$ Triton X-100. After blocking with 5\% skim milk for $60 \mathrm{~min}$, cells were incubated with antibody to $\gamma \mathrm{H} 2 \mathrm{~A} . \mathrm{X}$ (Millipore, Darmstadt, Germany) for $90 \mathrm{~min}$ at $37{ }^{\circ} \mathrm{C}$. The cells were then incubated with secondary antibody $\left(\mathrm{Cy}^{\mathrm{TM}} 3\right.$-conjugated AffiniPure $\mathrm{F}(\mathrm{ab})_{2}$ Fragment Goat Anti-Mouse IgG(H + L), Molecular Probes, Eugene, Oregon, USA) for $60 \mathrm{~min}$ at $37{ }^{\circ} \mathrm{C}$. Immunostained cells were observed by fluorescence microscopy in order to score $\gamma \mathrm{H} 2 \mathrm{~A} . \mathrm{X}$ foci. 


\section{Statistical analysis}

Mann-Whitney $U$-test, Kruskal-Wallis or Fisher's exact test were used to compare LINE-1 methylation levels between variables. A receiver operating characteristic (ROC) curve was created to evaluate the threshold of LINE-1 methylation level. Kaplan-Meier analysis and the log-rank test were used to evaluate differences in survival between patient groups. The prognostic significance of multiple variables was evaluated using a Cox proportional hazard regression model. Scores for $\gamma \mathrm{H} 2 \mathrm{~A} . \mathrm{X}$ foci were compared by Mann-Whitney $U$-test. All P-values shown are two-tailed, with $P<0.05$ taken as significant. All statistical analyses were performed with EZR (Saitama Medical Center, Jichi Medical University, Saitama, Japan), which is a graphical user interface for $\mathrm{R}$ (The R Foundation for Statistical Computing, Vienna, Austria, version 3.1.2). It is a modified version of $R$ commander (version 2.1-5) designed to add statistical functions that are frequently used in biostatistics [35].

\section{Results}

Tumor LINE-1 hypomethylation correlated with poor response to FOLFOX and with worse survival of advanced-stage CRC patients

LINE-1 methylation (median; 25th-75th percentile) in the primary tumor of advanced-stage CRC patients (47.4\%; 42.2-53.5\%) (Fig. 1; Additional file 3: Figure $\mathrm{S} 2 \mathrm{~A})$ was lower than in our previous study [22] of stage II and III CRC (84.7\%; 27.8-94.0\%). None of the patients in the present series was categorized as being in the high LINE-1 methylation (>84.3\%) group defined in our previous study [22]. No significant associations were found between LINE-1 methylation and clinicopathological

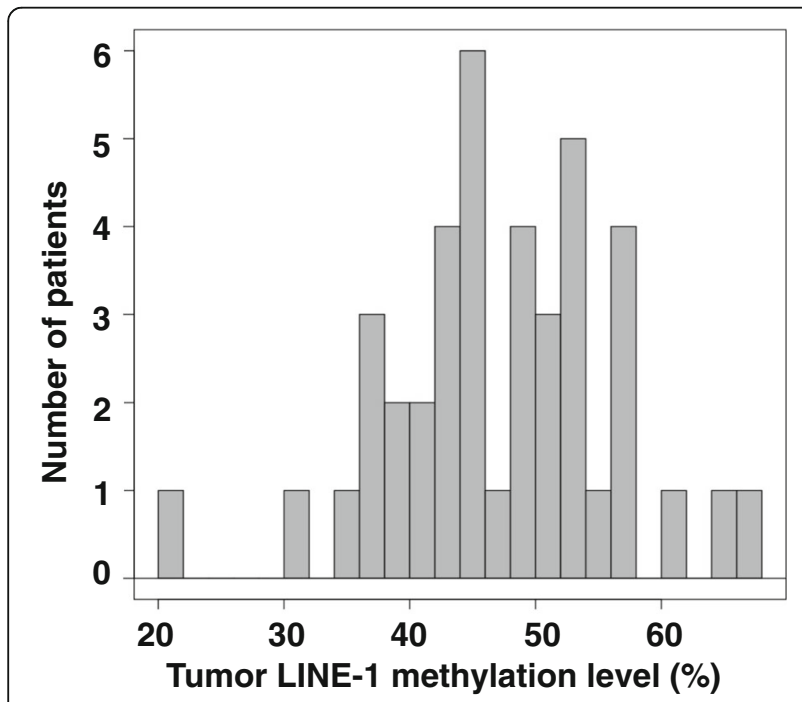

Fig. 1 Distribution of LINE-1 methylation level in the primary tumors of advanced-stage CRC. The median level of LINE-1 methylation was $47.4 \%$ characteristics including age, sex, primary tumor site, metastatic sites and prior treatment with 5-FU (Table 1). Higher LINE-1 methylation levels were associated with a trend for better clinical response to FOLFOX chemotherapy (Fig. 2; Additional file 3: Figure S2B), however this did not reach statistical significance $(P=0.18)$.

An ROC curve was constructed to assess response (complete response, partial response and stable disease) or no response (progressive disease) to FOLFOX chemotherapy according to tumor LINE-1 methylation (Fig. 3a). The cutoff value for LINE-1 methylation level was $51.7 \%$, as determined by the point yielding the greatest sum for sensitivity and specificity. The area under the curve (AUC) was 0.67 (95\% CI: 0.490.85). Tumor LINE-1 methylation levels were thus classified as high $(n=14)$ or low $(n=27)$ according to this cutoff value. No significant differences in clinical and histopathological characteristics were apparent between the high and low LINE-1 methylation patient groups (Additional file 4: Table S2). However, the PFS, OS and 5-year OPS were significantly worse in the low compared to high methylation group (PFS: median 6.6 vs. 9.4 months, $P=0.02$; OS: median 16.6 vs. 23.2 months, $P=0.01$; 5-year OPS: $4.1 \%$ vs. $28.6 \%$ ) (Fig. 3b, c). Multivariate analysis by COX proportional hazard model demonstrated that LINE-1 hypomethylation was an independent factor for poor prognosis $(P=0.018$; Table 2 , Additional file 5: Table S3, Additional file 6: Figure S3).

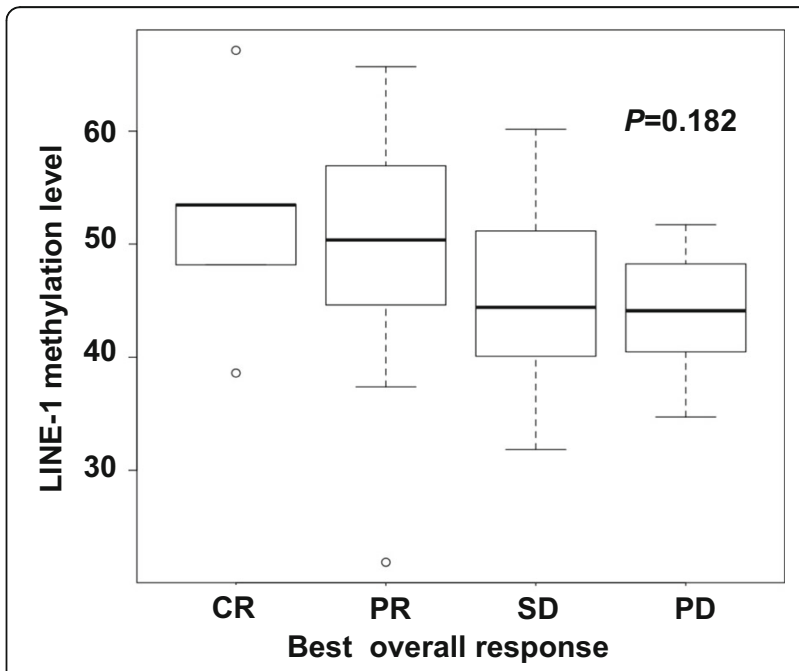

Fig. 2 Tumor LINE-1 methylation levels in CRC patient groups classified according to their best overall response. LINE-1 methylation levels are shown as the median and 25th-75th percentile. CR, complete remission; PR, partial remission; SD, stable disease; PD, progressive disease 


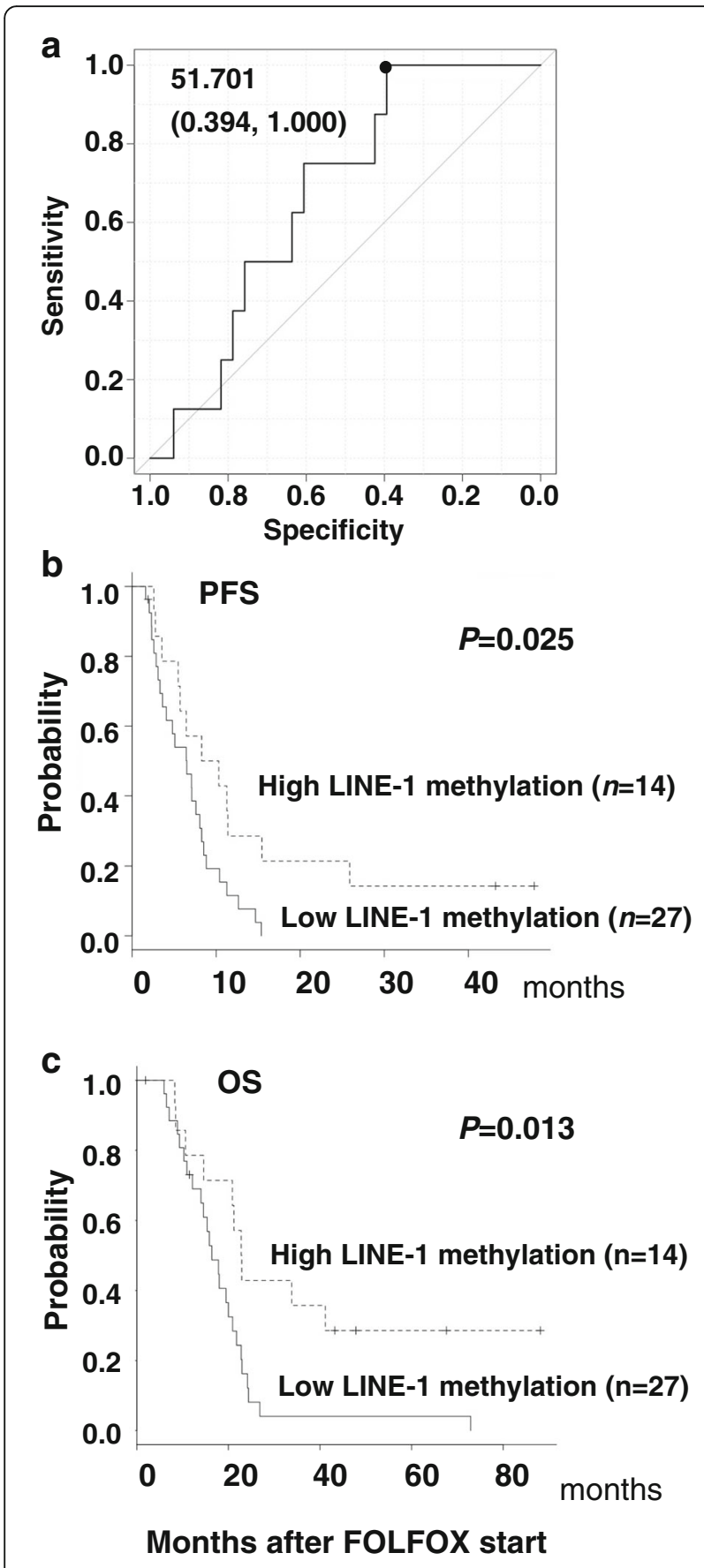

Fig. 3 Comparison of tumor LINE-1 methylation levels with the survival of advanced-stage CRC patients. a ROC curve was constructed according to response (CR, PR and $\mathrm{SD}$ ) or no response (PD) to the FOLFOX regimen. The cut-off value was determined as the point yielding the greatest sum of sensitivity and specificity for prediction of outcome. b, c Kaplan-Meier analysis of progression-free and overall survival of patient groups stratified according to their tumor LINE-1 methylation level. The log-rank test was used for each comparison and p-values are shown
Effects of oxaliplatin on LINE-1 activity and DNA damage in colon cancer cells

It was previously reported that SW480 and Caco2 cells show low LINE-1 methylation (55\%) and express LINE1, whereas HCT116 and RKO cells have higher LINE-1 methylation (65-90\%) and express little or no LINE-1 $[22,36]$. The effect of oxaliplatin on DNA stability in colon cancer cells with different levels of LINE-1 methylation was examined here using FAIRE. The baseline score for LINE-1 activity was higher in SW480 cells compared to HCT116, RKO and Caco2 cells, although the latter cell line also expressed LINE-1 (Fig. 4a; Additional file 7: Figure S4). Treatment of cells with $2 \mu \mathrm{M}$ oxaliplatin for $2 \mathrm{~h}$ decreased the LINE-1 score in HCT116 and RKO, but not in SW480 and Caco2 cells. This suggests that oxaliplatin stabilizes the DNA of colon cancer cells with high LINE-1 methylation by attenuating LINE-1 transposition activity.

Next, the level of DNA damage in cells following treatment with oxaliplatin was evaluated by scoring the number of $\gamma \mathrm{H} 2 \mathrm{~A}$.X foci. This value reflects the extent of DNA double-strand breaks. In the untreated condition, $\gamma \mathrm{H} 2 \mathrm{~A}$.X foci (median; range) were more frequently $(P<0.01)$ detected in LINE-1-expressing SW480 (3.37; 0-20) and Caco2 cells $(4.89 ; 0-40)$ than in LINE-1-non-expressing HCT116 (1.90; 0-20) and RKO cells (0.64; 0-12) (Fig. 4b). Treatment with $2 \mu \mathrm{M}$ oxaliplatin for $2 \mathrm{~h}$ increased the number of foci in all cell lines. The effect of oxaliplatin on SW480 and HCT116 cells was monitored for $2 \mathrm{~h}, 48 \mathrm{~h}$ and $120 \mathrm{~h}$. While the number of $\gamma \mathrm{H} 2 \mathrm{~A} . \mathrm{X}$ foci in SW480 cells increased progressively following treatment with oxaliplatin, the effect was transient in HCT116 (Fig. 4c). These results suggest the DNA in LINE-1-expressing cells such as SW480 is unstable and that treatment with oxaliplatin reinforces the DNA damage for an extended period over $120 \mathrm{~h}$.

\section{Combined effect of oxaliplatin and 5-FU on colon cancer cells}

The combined effect of oxaliplatin and 5-FU on DNA double-strand breaks was compared in LINE-1-expressing (SW480) and non-expressing (HCT116) cells (Fig. 5, Additional file 8: Figure S5). In SW480 cells, treatment with $2 \mu \mathrm{M} 5$-FU alone for $48 \mathrm{~h}$ increased the number of $\gamma \mathrm{H} 2 \mathrm{~A}$.X foci 1.28 -fold compared to untreated cells. Pretreatment of SW480 cells with $2 \mu \mathrm{M}$ oxaliplatin enhanced the effect of 5-FU between 1.49- and 1.92fold and this effect persisted for up to $120 \mathrm{~h}$. In HCT116 cells, treatment with $2 \mu \mathrm{M} 5$-FU alone for $48 \mathrm{~h}$ increased the number of $\gamma \mathrm{H} 2 \mathrm{~A}$.X foci 4.26 -fold compared to untreated cells $(P<0.0005)$. Pretreatment of HCT116 cells with $2 \mu \mathrm{M}$ oxaliplatin enhanced the effect of 5 -FU by 7.16 -fold $(P<0.0005)$, but no combined effect was found at $120 \mathrm{~h}$ after treatment. These results 
Table 2 Multivariate analysis for prognostic significance of clinicopathologic factors and tumor LINE-1 methylation in metastatic CRC

\begin{tabular}{|c|c|c|c|}
\hline Variables & Hazard ratio & $(95 \% \mathrm{Cl})$ & $p$-value \\
\hline Female & 0.85 & $(0.38-1.91)$ & 0.70 \\
\hline Older patients ${ }^{\mathrm{a}}$ & 0.63 & $(0.28-1.41)$ & 0.26 \\
\hline Rectum (vs. colon) & 0.84 & $(0.38-1.85)$ & 0.66 \\
\hline Well differentiation (vs. others) & 0.95 & $(0.26-3.41)$ & 0.94 \\
\hline Recurrence (vs. stage IV) & 0.81 & $(0.31-2.14)$ & 0.67 \\
\hline Multiple organs (vs. one organ) metastasis & 0.85 & $(0.34-2.08)$ & 0.72 \\
\hline Previous treatment with 5-FU & 1.62 & $(0.55-4.77)$ & 0.38 \\
\hline Second line (vs. first line) chemotherapy & 1.08 & $(0.37-3.11)$ & 0.89 \\
\hline LINE-1 hypomethylation ${ }^{b}$ & 2.74 & $(1.19-6.29)$ & 0.018 \\
\hline
\end{tabular}

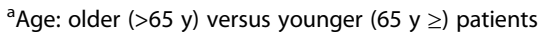

${ }^{b}$ The levels of tumor LINE-1 methylation were classified as hypomethylation versus hypermethylation based on the cutoff value (51.7\%) determined by the ROC curve (Fig. 3a)

indicate that the effects of sequential treatment with oxaliplatin and 5-FU, which mimic the clinically used FOLFOX regimen, depend on the level of LINE-1 methylation in colon cancer cells.

\section{Discussion}

Several previous studies have reported an association between tumor LINE-1 hypomethylation and worse survival of CRC patients from different clinical stages [19-21, 25-27]. We have also reported that 5-FU-based adjuvant chemotherapy appeared to benefit stage II and III CRC patients with low tumor LINE-1 methylation, but not those with high methylation [22]. In the present study we investigated LINE-1 methylation in the primary tumors of CRC patients with distant metastasis and/or recurrent tumors. We evaluated the prognostic relevance (PFS, OS, 5-year OPS) of LINE-1 methylation in patients who received chemotherapy with the combined oxaliplatin and 5-FU (FOLFOX) regimen. In agreement with the earlier studies cited above, tumor LINE-1 hypomethylation (i.e. below the cutoff value of $51.7 \%$ determined by ROC analysis) was associated with worse patient survival (Fig. 3b, c). In multivariate analysis, LINE-1 hypomethylation was shown to be an independent prognostic factor
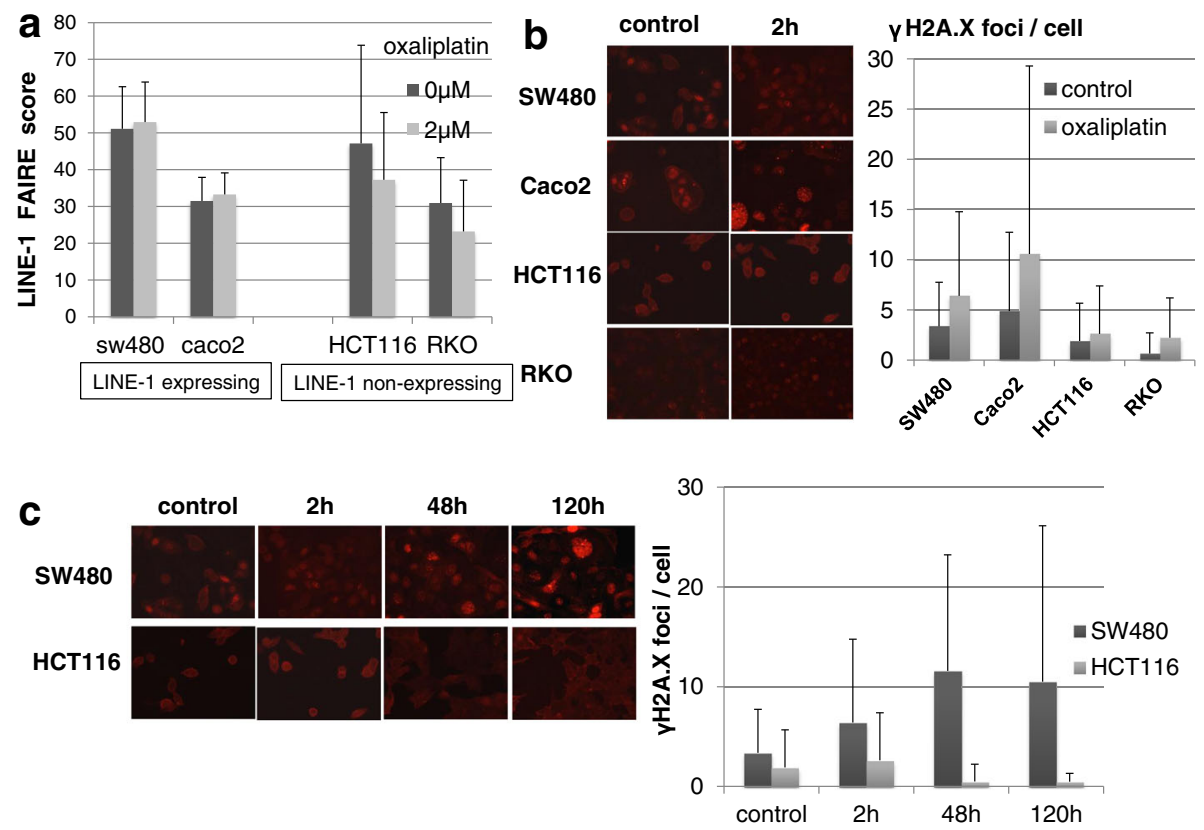

Fig. 4 Effects of oxaliplatin on LINE-1 activity and $\mathrm{YH} 2 \mathrm{~A}$.X foci in colon cancer cell lines. a Comparison of LINE-1 scores determined by FAIRE in LINE-1-expressing (SW480, Caco2) and non-expressing (HCT116, RKO) colon cancer cells that were treated with or without $2 \mu \mathrm{M}$ oxaliplatin for $2 \mathrm{~h}$. $\mathbf{b}$ Comparison of $\mathrm{YH} 2 \mathrm{~A}$.X foci in colon cancer cells treated with or without $2 \mu \mathrm{M}$ oxaliplatin for $2 \mathrm{~h}$. $\mathbf{c}$ Comparison of $\mathrm{YH} 2 \mathrm{~A}$.X foci between SW480 and HCT116 cells treated with or without $2 \mu \mathrm{M}$ oxaliplatin for 2, 48 and $120 \mathrm{~h}$, respectively 


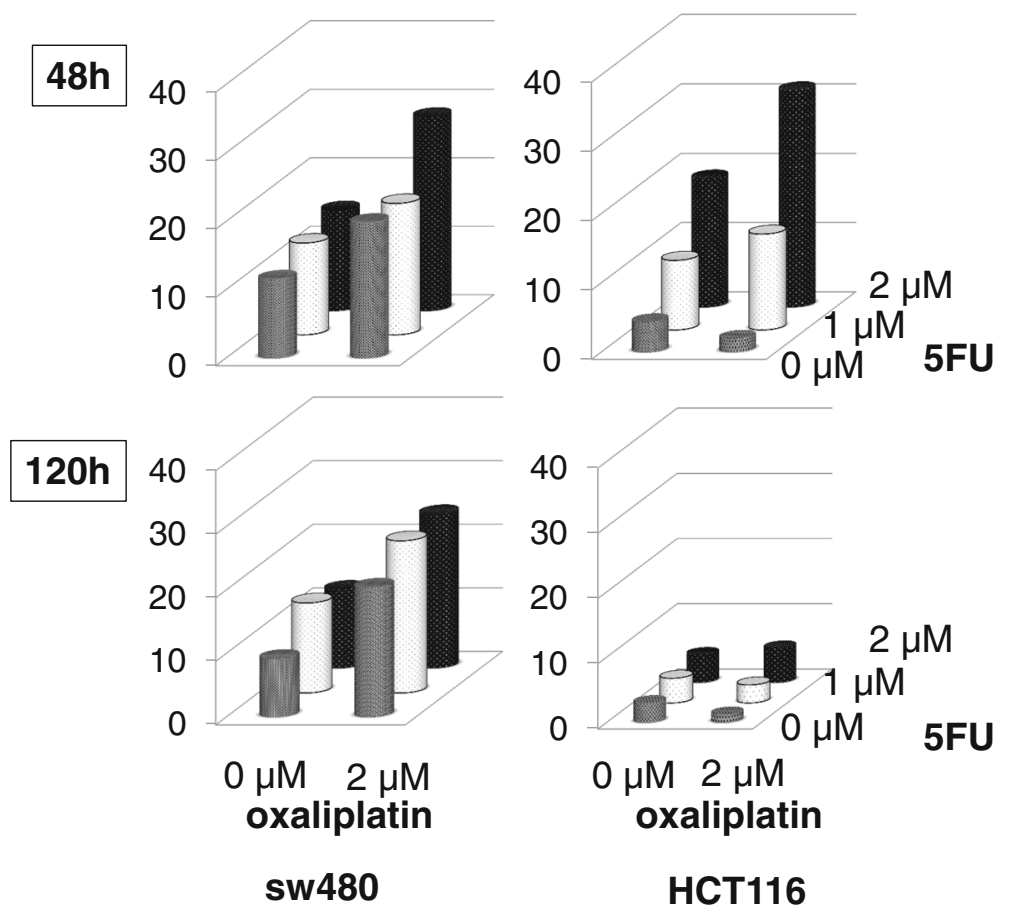

Fig. 5 Combined effect of oxaliplatin and 5-FU treatment on LINE-1 expressing and non-expressing colon cancer cells. SW480 (LINE-1 expressing) and HCT116 (LINE-1 non-expressing) cells were treated with oxaliplatin (2 $\mu \mathrm{M})$ alone, 5-FU (1 $\mu \mathrm{M}, 2 \mu \mathrm{M})$ alone, or a combination of both. For the combined treatment, the respective cells were treated sequentially with $2 \mu \mathrm{M}$ oxaliplatin for $2 \mathrm{~h}$ and then with 5 -FU (1 $\mu \mathrm{M}$ or $2 \mu \mathrm{M})$ for $48 \mathrm{~h}$ and $120 \mathrm{~h}$. $\mathrm{YH} 2 \mathrm{~A} . \mathrm{X}$ foci were measured following the respective treatments. Statistical differences between the data are shown in Additional file 4: Figure S2

in advanced-stage CRC. The significant difference in survival between patients with high and low tumor LINE-1 methylation levels, together with the trend for inverse association between response to chemotherapy and LINE-1 methylation (Fig. 2), suggest this marker could help to select advanced-stage CRC patients who may benefit from FOLFOX.

We previously reported that stage II and III CRC patients with high LINE-1 methylation in their primary tumor did not show a survival benefit from adjuvant chemotherapy with oral 5-FU [22]. This apparent discrepancy with the current results from advanced stage CRC patients may be due to the different stages of the patient cohorts, the different chemotherapy regimen used, as well as the different cutoff values used to define high and low LINE-1 methylation levels. In previous studies, LINE-1 methylation levels were divided into the three categories of high, intermediate and low [22, 37, 38]. In our earlier study [22], patients defined as having "high" ( $\geq 84 \%)$ methylation showed favorable prognosis but little response to oral 5-FU chemotherapy. The "intermediate" $(52-84 \%)$ and "low" (<52\%) methylation tumor groups are susceptible to metastasis, while the former group is sensitive but the latter insensitive to 5-FU-based chemotherapy. According to this categorization and based on the cutoff value (51.7\%) determined by the current ROC analysis (Fig. 3a), patients with high LINE-1 methylation as defined in the present study are included in the "intermediate" methylation group and there were no patients showing "high" methylation as defined above $(\geq 84 \%)$. This could explain why patients with higher LINE-1 methylation $(\geq 51.7 \%)$ showed better outcome following FOLFOX treatment compared to those with lower LINE-1 methylation $(<51.7 \%)$. Consistent with previous studies [37, 38], patients with low LINE-1 methylation responded poorly to FOLFOX. Aside from the different CRC clinical stages and chemotherapy regimens, the difference in cutoff value for LINE-1 methylation ( $84.3 \%$ vs. 51.7\%) between our previous [22] and present study does not allow direct comparison of the predictive value of LINE-1 methylation for response to 5-FU-based chemotherapy.

Previous [22, 37, 38] and present studies used DNA extracted from FFPE tumor tissues for bisulfite conversion and subsequent measurement of LINE-1 methylation. This may raise concern whether formalin fixation-associated DNA crosslinking and degradation will confound the quantitative PCR-based analysis of LINE-1 methylation. Therefore, this issue should be carefully addressed for clinical translation of LINE-1 methylation as a predictive biomarker in CRC. 
To investigate putative mechanistic links between tumor LINE-1 methylation and therapeutic effects of the FOLFOX regimen, we examined human colon cancer cell lines with different LINE-1 methylation and expression levels. DNA double-strand breaks were induced by sequential combination treatment with oxaliplatin and 5-FU, thus mimicking the clinical FOLFOX regimen. The DNA damaging effect of this combined treatment was more marked and persistent in LINE-1-expressing SW480 cells (lower methylation) compared to HCT116 cells with no LINE-1 expression (higher methylation; Fig. 4c). This observation is consistent with previous reports that the DNA of LINE-1-expressing cancer cells is unstable due to its retrotransposition ability and is therefore inherently susceptible to cytotoxic insults $[7,8]$. However, there is no direct evidence suggesting the correlation between LINE-1 expression and genomic instability. The different $\gamma \mathrm{H} 2 \mathrm{~A}$.X activity in different cell lines may be attributed to other sources such as intrinsic resistance (e.g., the ability of drug efflux) or the activities of DNA damage sensing and repair. The therapeutic effect observed in these colon cancer cells (Fig. 5) is inconsistent with the difference in outcome between advanced-stage CRC patients with high and low LINE-1 methylation levels treated with FOLFOX (Fig. 3). To address this discrepancy between clinical and in vitro findings, further investigations including verification of FFPE tissue samples as discussed above are needed on the expression of other known predictive biomarkers in experimental and clinical settings of combined oxaliplatin/5-FU regimens. These include the expression of thymidylate synthase [9] and dihydropyrimidine dehydrogenase [39] for 5-FU and excision-repair crosscomplementing-1 for oxaliplatin [10].

Methylation of genes other than LINE-1, including hMLH1, TFAP2E and SPARC, have been associated with resistance to chemotherapy [14, 40-42]. Based on such findings, a phase I clinical trial was undertaken to test the safety and efficacy of azacitidine, a demethylating agent, in combination with oxaliplatin for patients with advanced cancers (including CRC) that had relapsed or were refractory to any platinum therapy [13].

\section{Conclusions}

We have demonstrated that tumor LINE-1 hypomethylation is an independent marker of poor prognosis in advanced-stage CRC and may also be predictive of nonresponse to combination oxaliplatin/5-FU chemotherapy. Further prospective studies are required to establish whether tumor LINE-1 methylation can be a clinically useful prognostic and predictive biomarker for 5-FUbased chemotherapy in CRC patients. A critical issue will be to standardize the method for measuring tumor LINE1 methylation level and for determining the cutoff value.

\section{Additional files}

Additional file 1: Table S1. Primers and probes used for MethyLight assay, methylation-specific real-time PCR and FAIRE analysis of LINE-1. (DOCX $17 \mathrm{~kb}$ )

Additional file 2: Figure S1. The CPG sites in LINE-1 and design of primers used for methylation analysis. (PPTX $78 \mathrm{~kb}$ )

Additional file 3: Figure S2. (A) Distribution of LINE-1 methylation level measured by using the primer set (2) (Additional file 1: Table S1; Additional file 2: Figure S1) in the primary tumors of advanced-stage CRC. (B) Tumor LINE-1 methylation levels in CRC patient groups classified according to their best overall response. LINE-1 methylation levels are measured by using the primer set (2) (Additional file 1: Table S1; Additional file 2: Figure S1) and shown as the median and 25th-75th percentile. CR, complete remission; PR, partial remission; SD, stable disease; PD, progressive disease. (PPTX $240 \mathrm{~kb}$ )

Additional file 4: Table S2. Comparison of the levels of tumor LINE-1 methylation with the clinical and histopathological characteristics of advanced-stage CRC patients. (DOCX $28 \mathrm{~kb}$ )

Additional file 5: Table S3. Multivariate analysis for the prognostic significance of clinicopathologic factors and tumor LINE-1 methylation in metastatic CRC. (DOCX $27 \mathrm{~kb})$

Additional file 6: Figure S3. Influence of clinical and histopathological parameters and of tumor LINE-1 methylation levels on the OS of patients was analyzed by Kaplan-Meier analysis. Levels of tumor LINE-1 methylation were classified as high or low based on the cutoff value (51.7\%) determined by the ROC curve (Fig. 3a). (PPTX 664 kb)

Additional file 7: Figure S4. Comparison of LINE-1 scores determined by FAIRE in LINE-1-expressing (SW480, Caco2) and non-expressing (HCT116, RKO) colon cancer cells that were treated with or without $2 \mu \mathrm{M}$ oxaliplatin for 2 hrs. The primer set (2) (Additional file 1: Table S1) was used for FAIRE analysis. (PPTX $38 \mathrm{~kb}$ )

Additional file 8: Figure S5. Statistical comparison of data shown in Fig. 5. Mann-Whitney U-test was used for each comparison and p-values are shown. (PPTX $161 \mathrm{~kb})$

\section{Abbreviations}

5-FU: 5-fluorouracil; CRC: Colorectal cancer; FAIRE: Formaldehyde-assisted isolation of regulatory elements; FFPE: Formalin-fixed and paraffinembedded; LINE-1: Long interspersed nucleotide element-1; OPS: Overall probability of survival; OS: Overall survival; PFS: Progression-free survival; ROC: Receiver operating characteristic; YH2A.X: Phosphorylated histone H2A.X

\section{Acknowledgements}

We sincerely appreciate Dr. Kazuyuki Kawakami (Department of Chemotherapy and Palliative Care, Tokyo Women's Medical University) for his advice and expertise relating to this study and to Dr. Barry lacopetta (University of Western Australia) for critical reading and editing of this manuscript.

\section{Funding}

This work was supported in part by Grants-in-Aid for Scientific Research from the Japan Society for the Promotion of Science (the grant No. 23390321).

\section{Availability of data and materials}

All data of this study are available from the corresponding author (M. K.) upon request.

\section{Authors' contributions}

MK formulated the design and concept of the study, carried out the LINE-1 methylation analysis, formaldehyde-assisted isolation of regulatory elements assay, and measurement of phospho-histone H2A.X foci, and drafted the manuscript. MK, HB, TY, HT and TM collected clinical samples and helped to draft the manuscript. All authors read and approved the final manuscript.

\section{Competing interests}

The authors declare that they have no competing interests. 


\section{Consent for publication}

Not applicable.

\section{Ethics approval and consent to participate}

This study has been performed in accordance with the Declaration of Helsinki. Since tissues used in this study are from the patients diagnosed between 1999 and 2010, a written informed consent is available from most but not all patients. However in accordance with Japanese ethical guidelines and law, the study protocol was reviewed and approved by the Kanazawa University Medical Ethics Committee (the reference No. 269) and by the Ishikawa Prefectural Central Hospital Ethics Committee (the reference No. 374). All samples were anonymized before analysis was performed, to guarantee the protection of privacy.

\section{Author details}

'Department of General and Cardiothoracic Surgery, Graduate School of Medical Science, Kanazawa University, 13-1 Takara-machi, Kanazawa 920-8641, Japan. ²Department of Gastrointestinal Surgery, Ishikawa Prefectural Central Hospital, Kanazawa, Japan. ${ }^{3}$ Division of Translational and Clinical Oncology, Cancer Research Institute, Kanazawa University, Kanazawa, Japan

Received: 8 March 2016 Accepted: 29 November 2016 Published online: 12 December 2016

\section{References}

1. Torre LA, Bray F, Siegel RL, Ferlay J, Lortet-Tieulent J, Jemal A. Global Cancer Statistics, 2012. CA Cancer J Clin. 2015:65(2):87-108.

2. Edwards BK, Ward E, Kohler BA, Eheman C, Zauber AG, Anderson RN, Jemal A, Schymura MJ, Lansdorp-Vogelaar I, Seeff LC, et al. Annual report to the nation on the status of cancer, 1975-2006, featuring colorectal cancer trends and impact of interventions (risk factors, screening, and treatment) to reduce future rates. Cancer. 2010;116(3):544-73.

3. de Gramont A, Figer A, Seymour M, Homerin M, Hmissi A, Cassidy J, Boni C, Cortes-Funes $\mathrm{H}$, Cervantes A, Freyer $\mathrm{G}$, et al. Leucovorin and fluorouracil with or without oxaliplatin as first-line treatment in advanced colorectal cancer. J Clin Oncol. 2000;18(16):2938-47.

4. Goldberg RM, Sargent DJ, Morton RF, Fuchs CS, Ramanathan RK, Williamson SK, Findlay BP, Pitot HC, Alberts SR. A randomized controlled trial of fluorouracil plus leucovorin, irinotecan, and oxaliplatin combinations in patients with previously untreated metastatic colorectal cancer. J Clin Oncol. 2004;22(1):23-30.

5. Lao W, Grady MW. Epigenetics and colorectal cancer. Nat Rev Gastroenterol Hepatol. 2011;8(12):686-700.

6. Ji W, Hernandez R, Zhang XY, Qu GZ, Frady A, Varela M, Ehrlich M. DNA demethylation and rearrangements of chromosome 1. Mutat Res. 1997; 379(1):33-41.

7. Matsuzaki K, Deng G, Tanaka H, Kakar S, Miura S, Kim YS. The relationship between global methylation level, loss of heterozygosity, and microsatellite instability in sporadic colorectal cancer. Clin Cancer Res. 2005;11(24):8564-9.

8. Rodriguez J, Frigola J, Vendrell E, Risques RA, Fraga MF, Morales C, Moreno V, Esteller M, Capellà G, Ribas M, et al. Chromosomal instability correlates with genome-wide DNA demethylation in human primary colorectal cancers. Cancer Res. 2006;66(17):8462-8

9. Crea F, Nobili S, Paolicchi E, Perrone G, Napoli C, Landini I, Danesi R, Mini E. Epigenetics and chemoresistance in colorectal cancer: an opportunity for treatment tailoring and novel therapeutic strategies. Drug Resist Updat. 2011;14(6):280-96.

10. Alcindor T, Beauger N. Oxaliplatin: a review in the era of molecularly targeted therapy. Curr Oncol. 2011;18(1):18-25.

11. Arnould S, Hennebelle I, Canal P, Bugat R, Guichard S. Cellular determinants of oxaliplatin sensitivity in colon cancer cell lines. Eur J Cancer. 2003;39(1):112-9.

12. Stewart DJ. Mechanisms of resistance to cisplatin and carboplatin. Crit Rev Oncol Hematol. 2007;63(1):12-31.

13. Tsimberidou AM, Said R, Culotta K, Wistuba I, Jelinek J, Fu S, Falchook G, Naing A, Piha-Paul S, Zinner R, et al. Phase I study of azacitidine and oxaliplatin in patients with advanced cancers that have relapsed or are refractory to any platinum therapy. Clin Epigenetics. 2015;7(1):29.

14. Plumb JA, Strathdee G, Sludden J, Kaye SB, Brown R. Reversal of drug resistance in human tumor xenografts by 2'-deoxy-5-azacytidine-induced demethylation of the hMLH1 gene promoter. Cancer Res. 2000;60(21):6039-44.
15. Lander ES, Linton LM, Birren B, Nusbaum C, Zody MC, Baldwin J, Devon K, Dewar K, Doyle M, FitzHugh W, et al. Initial sequencing and analysis of the human genome. Nature. 2001;409(6822):860-921.

16. Deininger PL, Moran JV, Batzer MA, Kazazian Jr HH. Mobile elements and mammalian genome evolution. Curr Opin Genet Dev. 2003;13(6):651-8.

17. Ding W, Lin L, Chen B, Dai J. L1 elements, processed pseudogenes and retrogenes in mammalian genomes. IUBMB Life. 2006;58(12):677-85.

18. Weisenberger DJ, Campan M, Long TI, Kim M, Woods C, Fiala E, Ehrlich M, Laird PW. Analysis of repetitive element DNA methylation by MethyLight. Nucleic Acids Res. 2005:33(21):6823-36.

19. Rhee YY, Kim MJ, Bae JM, Koh JM, Cho NY, Juhnn YS, Kim D, Kang GH. Clinical outcomes of patients with microsatellite-unstable colorectal carcinomas depend on L1 methylation level. Ann Surg Oncol. 2012;19(11):3441-8.

20. Ahn JB, Chung WB, Maeda O, Shin SJ, Kim HS, Chung HC, Kim NK, Issa JP. DNA methylation predicts recurrence from resected stage III proximal colon cancer. Cancer. 2011;117(9):1847-54.

21. Ogino S, Nosho K, Kirkner GJ, Kawasaki T, Chan AT, Schernhammer ES, Giovannucci EL, Fuchs CS. A cohort study of tumoral LINE-1 hypomethylation and prognosis in colon cancer. J Natl Cancer Inst. 2008; 100(23):1734-8.

22. Kawakami K, Matsunoki A, Kaneko M, Saito K, Watanabe G, Minamoto T. Long interspersed nuclear element-1 hypomethylation is a potential biomarker for the prediction of response to oral fluoropyrimidines in microsatellite stable and CpG island methylator phenotype-negative colorectal cancer. Cancer Sci. 2011;102(1):166-74.

23. Matsunoki A, Kawakami K, Kotake M, Kaneko M, Kitamura H, Ooi A, Watanabe G, Minamoto T. LINE-1 methylation shows little intra-patient heterogeneity in primary and synchronous metastatic colorectal cancer. BMC Cancer. 2012;12:574.

24. Murata A, Baba Y, Watanabe M, Shigaki H, Miyake K, Ishimoto T, Iwatsuki M, Iwagami S, Sakamoto Y, Miyamoto Y, et al. Methylation levels of LINE-1 in primary lesion and matched metastatic lesions of colorectal cancer. $\mathrm{Br}$ J Cancer. 2013;109(2):408-15.

25. Sunami E, de Maat M, Vu A, Turner RR, Hoon DSB. LINE-1 Hypomethylation during primary colon cancer progression. PLoS One. 2011;6(4):e18884.

26. Mima K, Nowak JA, Qian ZR, Cao Y, Song M, Masugi Y, Shi Y, da Silva A, Gu M, Li W, et al. Tumor LINE-1 methylation level and colorectal cancer location in relation to patient survival. Oncotarget. 2016. doi:10.18632 oncotarget.10398. E-Pub

27. Lou Y-T, Chen C-W, Fan Y-C, Chang W-C, Lu C-Y, Wu I-C, Hsu W-H, Huang C-W, Wang J-Y. LINE-1 methylation status correlates significantly to posttherapeutic recurrence in stage III colon cancer patients receiving FOLFOX-4 adjuvant chemotherapy. PLoS One. 2015;10(4):e0123973.

28. Kanemitsu Y, Kato T, Shimizu Y, Inaba Y, Shimada Y, Nakamura K, Sato A Moriya Y, Colorectal Cancer Study Group (CCSG) of Japan Clinical Oncology Group. A randomized phase II/III trial comparing hepatectomy followed by mFOLFOX6 with hepatectomy alone as treatment for liver metastasis from colorectal cancer: Japan Clinical Oncology Group Study JCOG0603. Jpn J Clin Oncol. 2009:39(6):406-9.

29. Eisenhauer EA, Therasse P, Bogaerts J, Schwartz LH, Sargent D, Ford R, Dancey J, Arbuck S, Gwyther S, Mooney M, et al. New response evaluation criteria in solid tumours: revised RECIST guideline (version 1.1). Eur J Cancer. 2009:45(2):228-47.

30. Raymond E, Buquet-Fagot C, Djelloul S, Mester J, Cvitkovic E, Allain P, Louvet C, Gespach C. Antitumor activity of oxaliplatin in combination with 5-fluorouracil and the thymidylate synthase inhibitor AG337 in human colon, breast and ovarian cancers. Anticancer Drugs. 1997;8(9):876-85.

31. Eads CA, Danenberg KD, Kawakami K, Saltz LB, Blake C, Shibata D, Danenberg PV, Laird PW. MethyLight: a high-throughput assay to measure DNA methylation. Nucleic Acids Res. 2000;28:E32.

32. lacopetta B, Grieu F, Phillips M, Ruszkiewicz A, Moore J, Minamoto T, Kawakami K. Methylation levels of LINE-1 repeats and CpG island loci are inversely related in normal colonic mucosa. Cancer Sci. 2007:98(9):1454-60.

33. Giresi PG, Kim J, McDaniell RM, lyer VR, Lieb JD. FAIRE (FormaldehydeAssisted Isolation of Regulatory Elements) isolates active regulatory elements from human chromatin. Genome Res. 2007:17(6):877-85.

34. De Cecco M, Criscione SW, Peckham EJ, Hillenmeyer S, Hamm EA, Manivannan J, Peterson AL, Kreiling JA, Neretti N, Sedivy JM. Genomes of replicatively senescent cells undergo global epigenetic changes leading to gene silencing and activation of transposable elements. Aging Cell. 2013; 12(2):247-56. 
35. Kanda Y. Investigation of the freely-available easy-to-use software "EZR" (Easy R) for medical statistics. Bone Marrow Transplant. 2013;48(3):452-8.

36. Link A, Balaguer F, Shen Y, Lozano JJ, Leung HC, Boland CR, Goel A. Curcumin modulates DNA methylation in colorectal cancer cells. PLoS One. 2013;8(2):e57709.

37. Ogino S, Nishihara R, Lochhead P, Imamura Y, Kuchiba A, Morikawa T, Yamauchi M, Liao X, Qian ZR, Sun R, et al. Prospective study of family history and colorectal cancer risk by tumor LINE-1 methylation level. J Natl Cancer Inst. 2013;105(2):130-40.

38. Inamura K, Yamauchi M, Nishihara R, Lochhead P, Qian ZR, Kuchiba A, Kim SA, Mima K, Sukawa Y, Jung S, et al. Tumor LINE-1 methylation level and microsatellite instability in relation to colorectal cancer prognosis. J Natl Cancer Inst. 2014;106(9):dju195.

39. Fischel JL, Formento P, Ciccolini J, Rostagno P, Etienne MC, Catalin J, Milano G. Impact of the oxaliplatin-5 fluorouracil-folinic acid combination on respective intracellular determinants of drug activity. Br J Cancer. 2002;86(7):1162-8.

40. Chekhun VF, Lukyanova NY, Kovalchuk O, Tryndyak VP, Pogribny IP. Epigenetic profiling of multidrug-resistant human MCF-7 breast adenocarcinoma cells reveals novel hyper- and hypomethylated targets. Mol Cancer Ther. 2007;6(3):1089-98.

41. Coppedè F, Lopomo A, Spisni R, Migliore L. Genetic and epigenetic biomarkers for diagnosis, prognosis and treatment of colorectal cancer. World J Gastroenterol. 2014;20(4):943-56.

42. Chang X, Monitto CL, Demokan S, Kim MS, Chang SS, Zhong X, Califano JA Sidransky D. Identification of hypermethylated genes associated with cisplatin resistance in human cancers. Cancer Res. 2010;70(7):2870-9.

\section{Submit your next manuscript to BioMed Central} and we will help you at every step:

- We accept pre-submission inquiries

- Our selector tool helps you to find the most relevant journal

- We provide round the clock customer support

- Convenient online submission

- Thorough peer review

- Inclusion in PubMed and all major indexing services

- Maximum visibility for your research

Submit your manuscript at www.biomedcentral.com/submit 